\title{
Erratum to: Quality assessment of estrogen receptor and progesterone receptor testing in breast cancer using a tissue microarray-based approach
}

\author{
T. J. A. Dekker ${ }^{1,2}$ - S. ter Borg ${ }^{3}$ - G. K. J. Hooijer ${ }^{3}$ S. L. Meijer ${ }^{3}$. \\ J. Wesseling ${ }^{4}$ J. E. Boers ${ }^{5}$ E. Schuuring ${ }^{6}$ J. Bart ${ }^{5,6} \cdot$ J. van Gorp ${ }^{7}$. \\ P. Bult ${ }^{8}$ - S. A. Riemersma ${ }^{9}$ - C. H. M. van Deurzen ${ }^{10}$ - H. F. B. M. Sleddens ${ }^{10}$. \\ W. E. Mesker ${ }^{2}$ J. R. Kroep ${ }^{1}$ V. T. H. B. M. Smit ${ }^{11}$ - M. J. van de Vijver ${ }^{3}$
}

Published online: 24 June 2015

(c) Springer Science+Business Media New York 2015

\section{Erratum to: Breast Cancer Res Treat DOI 10.1007/s10549-015-3444-x}

Unfortunately, in the original publication of the article, the abstract has an oversight. The authors regret for this error.

The sentence in the abstract that reads as "Sensitivity and specificity of individual laboratories for testing estrogen receptor expression were high, with an overall sensitivity of 99.7 and $95.4 \%$, respectively", should read as "Sensitivity and specificity of individual laboratories for testing estrogen receptor expression were high, with an overall sensitivity and specificity of 99.7 and $95.4 \%$, respectively".
The online version of the original article can be found under doi:10.1007/s10549-015-3444-x.

\footnotetext{
M. J. van de Vijver

m.j.vandevijver@amc.uva.nl

T. J. A. Dekker

T.J.A.Dekker@lumc.nl

1 Department of Medical Oncology, Leiden University Medical Center, Leiden, The Netherlands

2 Department of Surgery, Leiden University Medical Center, Leiden, The Netherlands

3 Department of Pathology, Academic Medical Center, Amsterdam, The Netherlands

4 Department of Pathology and Division of Molecular Pathology, Netherlands Cancer Institute, Amsterdam, The Netherlands

5 Department of Pathology, Isala, Zwolle, The Netherlands
}

6 Department of Pathology, University of Groningen, University Medical Center Groningen, Groningen, The Netherlands

7 Department of Pathology, Diakonessenhuis, Utrecht, The Netherlands

8 Department of Pathology, Radboud University Medical Center, Nijmegen, The Netherlands

9 Department of Pathology, Laboratory of Pathology East Netherlands, Hengelo, The Netherlands

10 Department of Pathology, Erasmus University Medical Center, Rotterdam, The Netherlands

11 Department of Pathology, Leiden University Medical Center, Leiden, The Netherlands 